\title{
The integration of family planning and genitourinary medicine services
}

\author{
Integrate - 'to complete (imperfect thing) by addition of parts'(Oxford English Dictionary).
}

From a client care centred perspective, traditional family planning and genitourinary medicine (GUM) services are in many ways imperfect as neither deals with the broad spectrum of sexual health needs in an holistic way. Client centred care focuses on equity of access, the provision of quality care, appropriate referral and management and achieving client satisfaction. Is the solution an extension of the recent trend of traditionally separate disciplines sharing clinical space, or is a more fundamental examination of clients' needs required if we are to address the sexual health needs of $21^{\text {st }}$ Century men and women?

The integration of services is a complex process; identifying and understanding the similarities in relation to client care is important and is perhaps the easy part of the equation. In contrast, the origins and subsequent evolution of the specialities of GUM and family planning are, in many ways, worlds apart. This means we have to consider several contrasting elements - gynaecologists and physicians; sessional staff and full time staff; preventative medicine and managing disease, and the role of the doctor and of the nurse. Thus we have, at this moment in time, two specialities that are in many ways doing similar work and have similar aims for client care, but which work in different ways. The challenges for integration lie within ourselves, not with the medical problems or with the clients.

For very good reasons GUM services developed along a medical model within hospitals and later within the NHS at its inception, initially to meet the needs of men returning from the army with venereal diseases at the end of the Great War; the emphasis being to provide confidential services free of charge. It is interesting that an early recommendation from the medical establishment on the prevention of the spread of STIs was to detain some women with STIs, not men, as hospital inpatients. Women's rights campaigners successfully changed opinion on this matter. Family planning services evolved from female empowerment and, to begin with, had only limited support from much of the medical profession. The latter deemed the field too 'social' for medical responsibility. Family planning services only became freely available to all in the mid 1970s when FPA clinics were absorbed into the NHS and primary care providers started to receive remuneration for providing contraceptive care.

When considering the integration of family planning and sexual health services, it is important to remember the breadth of providers involved - pharmacists (condoms, pregnancy tests, female barriers, Persona and, in the future, emergency contraception), obstetricians and gynaecologists, midwives, health visitors and health promotion staff, as well as doctors and nurses working in family planning services and GUM services in acute and community trusts and in primary care. Unlike in family planning, in GUM specific legislation exists to protect confidentiality. However, in practice, most family planning and GUM services work to similar standards.

Until the mid 1980's and the allocation of now huge budgets for HIV research, prevention and treatment, both family planning and GUM services could be considered 'Cinderella' specialities. Whilst GUM services have been able to undergo some expansion and development using 'HIV funding', there has been 'creative' use of funding for a wider range of sexual health and education services by only a few health authorities. Drug costs aside, the disparity between well staffed, well appointed HIV units and cash starved family planning services struggling with recruitment and retention problems, has undoubtedly led to inequality and different expectations between groups of practitioners whose areas of work overlap. Close working relationships or integrated services have mostly established themselves in areas where one service had never developed, or where one had been without leadership for a while or was undergoing major change as a result of health service reconfigurations.

Another historical obstacle to integration has been the different training routes undertaken by both doctors and nurses in family planning and GUM. The previous lack of formalised specialist training schemes in reproductive health care further hampered the opportunities for shared training.

The requirement for GUM trainees to obtain the DFFP, and the inclusion of significant modules of GUM in both SpR training in community gynaecology and career grade training in reproductive health care, are certainly moves in the right direction. Nevertheless, there remains a need for basic level training in GUM both for doctors already experienced in providing contraceptive and reproductive health care, and also those undertaking basic training. The time seems right for the development of recognised training combining theoretical and practical experience in the diagnosis and management of STIs, as exists in family planning in the form of the DFFP. Many skills are shared already e.g. the ability to talk about sex and perform genital examinations in a sympathetic way, but work is needed in relation to the pathology of STIs and partner notification. There needs to be discussion to ensure that all parties - family planning, GUM and client advocates - are comfortable that, when required, the confidentiality of the client is protected. With more training experienced family planning trained doctors would be able to provide a broader range of sexual health care to their clients and would have a clearer understanding of referral patterns and services provided in GUM clinics. This would also allow more innovative approaches to providing appropriate sexual health care to populations, wherever, as individuals, they access healthcare; unlike the current situation where the care received varies depending on which service or whom the client accesses.

Work on the content of such a training module could precipitate discussions about the skills needed to provide STI screening e.g. the role of microscopy in the diagnosis of STIs in men and women. Guidelines could also be developed about staff roles and referral patterns within different types of sexual health care provision. Perhaps such close working could also extend to developing the GUM component of the MFFP examination so that membership of the Faculty of Family Planning and Reproductive Health Care became recognised as the postgraduate qualification for specialists in women's sexual health? This then begs the question: is the diagnosis and management of sexually transmitted infections in women part of gynaecology, in its broadest sense, or a separate speciality? 
Common core training elements do not obviate the need for specialists in either reproductive health care or GUM able to provide specialist care, to train others and to play a strategic role in the development of sexual health services.

The interface between primary care and specialist services is crucial to the integration of reproductive and sexual health services with the development of guidelines about what to refer, who to refer and how to ensure appropriate referrals. The ability to make appointments with specialist services directly from the primary care setting via a computerised booking system that also integrates referral protocols (booked admission projects) might reduce the 'drop out' rate between first consultation and GUM specialist. Provision of facilities to diagnose and treat STIs among family planning clinic attenders in the same building has been shown to increase the likelihood of re-attendance and successful treatment.

Existing providers of family planning and GUM services in hospital, community and primary care settings have a great deal to learn from each other about access to services and skill mix. The enhanced role of nurses in contraceptive provision as clinical nurse specialists in family planning services and as practice nurses in primary care can be, and in some cases has been, applied successfully to the GUM settings with nurse-led clinics and triage by experienced senior nurses in walk-in clinics. Evidence from work with young people shows that clients want to see someone who is friendly and competent, and that they are happy to see nurses alone if all their needs can be met.

In integrating family planning and GUM services balances have to be struck between walk-in and appointment clinics, the timing and location of clinical services and the availability of consultant opinion, all of which must reflect client needs. A pragmatic approach to the range of STI screening services offered enables peripheral community family planning clinics and GP surgeries to screen people who would not otherwise take up opportunities for screening. Referral to a larger unit for full screening and partner notification is then an option, though many conditions can be managed entirely in the community. Is it better to have, for instance, much of the 'at risk' population screened for Chlamydia, or a small minority screened for everything? In times of limited resources there is therefore a potential conflict between the needs of the individual and those of the population.

Integrating family planning and GUM services offers the potential to develop the range of sexual health services that men and women want to use as clients in $21^{\text {st }}$ Century. The provision of acceptable services is the only way to address current sexual health issues and consultation with 'consumers' is vital. Many clients do not understand the traditional divisions between services; health care professionals need to follow their lead to avoid the psychiatric definition of integration - 'the combination of diverse elements of perception' (Oxford English Dictionary)

Christopher Wilkinson, MBBS, MFFP Consultant in Women's Sexual Health; Honorary Senior Lecturer in Gynaecology and Genitourinary Medicine King's College Hospital, London SE5 9RS, UK.

Naomi Hampton, BSc, MRCOG, MFFP Consultant in Reproductive Health Care and Community Gynaecology Enfield Community Care NHS Trust, UK

Caroline Bradbeer, MBBS, FRCP Consultant Physician Genitourinary Medicine, St Thomas' Hospital, London, UK 